Research Paper

\title{
Towards mass production of a spherical montmorillonite@covalent organic framework@gold nanoparticles heterostructure as a high-efficiency catalyst for reduction of methylene blue
}

\author{
Fushuai Wang ${ }^{\mathrm{a}}$, Fei Pan ${ }^{\mathrm{b}}$, Shunyang Yu ${ }^{\mathrm{b}, \mathrm{c}}$, Dawei Pan ${ }^{\mathrm{b}, \mathrm{c}}$, Peiqing Zhang ${ }^{\mathrm{a}, *}$, Ning Wang ${ }^{\mathrm{b}, \mathrm{c}, * *}$ \\ ${ }^{\text {a }}$ Yantai University, Yantai, Shandong Province 264005, PR China \\ ${ }^{\mathrm{b}}$ CAS Key Laboratory of Coastal Environmental Processes and Ecological Remediation, Research Center for Coastal Environmental Engineering and Technology of \\ Shandong Province, Shandong Key Laboratory of Coastal Environmental Processes, YICCAS, Yantai Institute of Coastal Zone Research, Chinese Academy of Sciences, \\ Yantai, Shandong Province 264003, PR China \\ ${ }^{\mathrm{c}}$ Center for Ocean Mega-Science, Chinese Academy of Sciences, 7 Nanhai Road, Qingdao 266071, PR China
}

\section{A R T I C L E I N F O}

\section{Keywords:}

Gold nanoparticles

Covalent organic framework

Microspheres

Catalytic reduction

Methylene blue

\begin{abstract}
A B S T R A C T
Gold nanoparticles (Au NPs) supported by a covalent organic framework (COF) exhibited a remarkably catalytic performance towards organic pollutants, while the application is limited by the recovery and reuse from the mixture due to the size of Au NPs@COF at the nanoscale. Herein, Au NPs were in situ reduced on the surface of sulfhydryl groups functionalized new COF with $\mathrm{NaBH}_{4}$, followed by the deposition on polydopamine modified micron-sized porous spherical montmorillonite (SMt) to construct a spherical SMt@COF@Au NPs heterostructure. SMt was used here due to its excellent adsorption ability towards methylene blue (MB), increasing the concentration of MB near COF@Au NPs, thus accelerating the catalytic reaction. Hence, the optimized apparent reduction rate constant $\left(K_{\mathrm{app}}\right)$ and turnover frequency (TOF) in our experiment could reach $8.32 \mathrm{~min}^{-1}$ and 3.2 $\times 10^{5} \mathrm{~h}^{-1}$, respectively, at $40{ }^{\circ} \mathrm{C}$ and $\mathrm{pH}$ values of 10 , which is much better than the reported. Furthermore, micro-sized SMt with self-sedimentary property could be easily recovered from the mixture without any devices involved. More importantly, the granular micro-sized morphology of the SMt-based heterostructure could facile transport on the product line and reduce the fouling, facilitating mass production COF@Au NPs based catalyst for application.
\end{abstract}

\section{Introduction}

Heterogeneous catalysts have attracted great attention in the areas of science and industry due to their excellent physical and chemical properties (Gao et al., 2020; Liao et al., 2019; Wang and Zhuang, 2019). Nanomaterials such as metal oxides (Cao et al., 2020; Das et al., 2019), clay materials (Arora et al., 2018; Joseph et al., 2019; Peng et al., 2019; Zhang et al., 2018), carbon materials (Qin et al., 2019a; Qin et al., 2019b), and metal-organic frameworks (Gao et al., 2019) were commonly used as carriers due to their large special surface areas to provide more active sites. Recently, the combination of two nanoparticles, covalent organic framework (COF) and ultrafine noble metal nanoparticles (average diameter of less than $5 \mathrm{~nm}$ ), as a nanocomposite catalyst represents the type of the most investigated heterogeneous catalytic system because of the unique structure and property of COF to control the size of noble metal nanoparticles and the excellent organic pollutants adsorption/electron transfer/products desorption ability for catalytic reduction of organic pollutants (Cao et al., 2017; Zhang et al., 2020c). However, the synthesized COF/ultrafine noble metal nanoparticles nanocomposites are in favor of dispersion in the solution, while it is difficult to separate from the mixture and reuse even through highspeed centrifugation, heavily increasing the cost of toxic organic pollutants treatment.

Considered this troublesome, the magnetic carrier could be a good choice (Yang et al., 2020). Zheng et al. reported the synthesized $\mathrm{Fe}_{3} \mathrm{O}_{4}$ supported COF/gold metal nanoparticles (Au NPs) (Xu et al., 2020). Hou

\footnotetext{
* Corresponding author.

** Corresponding author at: CAS Key Laboratory of Coastal Environmental Processes and Ecological Remediation, Research Center for Coastal Environmental Engineering and Technology of Shandong Province, Shandong Key Laboratory of Coastal Environmental Processes, YICCAS, Yantai Institute of Coastal Zone Research, Chinese Academy of Sciences, Yantai, Shandong Province 264003, PR China.

E-mail addresses: zhangpqytu@126.com (P. Zhang),nwang@yic.ac.cn (N. Wang).
} 
et al. reported the construction of core-shell structured $\mathrm{CuFe}_{2} \mathrm{O}_{4} / \mathrm{Ag}$ NPs/COF (Hou et al., 2020). Although magnetic separation can be used to recover these prepared catalysts, the usage of a great number of magnetic equipment is not in favor of the application. Compared with these carriers with nano-size, the porous micro-size carrier assembled by nanomaterials can also provide a large surface area for supporting noble metal nanoparticles. In our previous work, porous spherical montmorillonite (SMt) prepared with the granulation of Mt. through spraydrying technology owning the characteristics of large surface area, strong adsorption ability, and outstanding acid-base stability (Wang et al., 2020; Xiao et al., 2018). Different from the pristine Mt. with the strong hydratability and difficulty to subside in the aqueous solution, the granular compacted SMt microsphere could easily self-sedimentary in the mixture (Wang et al., 2019).

The catalytic transformation occurred on the surface of the catalyst including a complex adsorption-transformation-desorption procedure, where the design of heterogeneous catalyst structure regulates the interaction between the substrates and catalysts, further influencing the catalytic performance (Iqbal et al., 2018). Herein, ultrafine gold metal nanoparticles (Au NPs) were in situ reduced on the surface of sulfhydryl groups functionalized new $\mathrm{COF}$ with $\mathrm{NaBH}_{4}$, followed by the deposition on polydopamine modified micron-sized porous SMt to construct a spherical SMt@COF@Au NPs heterostructure. The resultant heterostructure was examined as a heterogeneous catalyst for catalytic reduction of methylene blue (MB) in the presence of $\mathrm{NaBH}_{4}$. The influences of concentration of catalyst, reaction temperature, and $\mathrm{pH}$ value on catalytic activity were systematically investigated. Recyclability was further studied. The self-sedimentary granularSMt@COF@Au NPs heterostructure is not only facile recovery from the mixture without any devices involved but also facilitates the mass production of these types of heterogeneous catalysts due to the improvement of transport and the reduction of fouling in the product-line compared with other amorphous substrates. To the best of our knowledge, this is the first report of fabricated a granular porous micro size carrier supported COF@Au NPs heterostructure as a catalyst for reduction of organic pollutants, successfully solving an engineering problem via a physical method.

\section{Experimental section}

\subsection{Chemical and materials}

2, 4, 6-trihydroxybenzene-1, 3, 5-tricarbaldehyde and 2, 5-Diamino1, 4-benzenedithiol dihydrochloride with purity above $98.0 \%$ were purchased from Jilin Chinese Academy of Sciences-Yanshen Technology Co., China. Other reagents and chemicals including 1, 4-Dioxane $(\geq$ $99.0 \%$ ), Mesitylene ( $\geq 98.0 \%$ ), sodium borohydride $\left(\mathrm{NaBH}_{4}, \geq 99.0 \%\right)$, dopamine hydrochloride ( $\geq 98.0 \%$ ), acetic acid ( $\geq 98.0 \%$ ), methylene blue (MB, $\geq 99.0 \%$ ), and montmorillonite were obtained from Sinopharm Chemical Reagent Co. Ltd., China. All the reagents were of analytically pure grade and used without any further purification. The structures and properties of the prepared samples, including COF-SH, COF@Au NPs, and SMt@COF@Au NPs heterostructure, was characterized by scanning electron microscopy (SEM, Jeol S4800), transmission electron microscopy (TEM, JEM 2200), X-ray photoelectron spectroscopy (XPS, Thermo Fisher, ${ }^{13} \mathrm{C}$ CP/MAS NMR (JNM-ECZ600R), X-ray diffraction (XRD, Bruker D8-Advantage powder diffractometer), surface area and pore analyzer (Micromeritics ASAP 2020), and inductively coupled plasma mass spectrometry (ICP-MS, Perkin Elmer ELAN DRC II), respectively. The catalytic performance of the prepared samples was evaluated by a UV-vis spectrometer (PERSEE UT 1810).

\subsection{Synthesis of COF-SH}

The synthesis of COF-SH was performed via the condensation of 2, 4, 6-trihydroxybenzene-1, 3, 5-tricarbaldehyde and 2, 5-Diamino-1, 4- benzenedithiol dihydrochloride. Typically, 2, 4, 6-trihydroxybenzene-1, 3, 5-tricarbaldehyde (25.21 mg, $0.12 \mathrm{mmol}$ ) and 2, 5-Diamino-1, 4benzenedithiol dihydrochloride $(44.11 \mathrm{mg}, 0.18 \mathrm{mmol})$ were mixed with $1.1 \mathrm{~mL}$ of a 3:7:1 $\mathrm{v} / \mathrm{v} / \mathrm{v}$ solution of 1 , 4-Dioxane/Mesitylene/ $6 \mathrm{M}$ aqueous acetic acid. After frozen in a liquid nitrogen bath and three successive freeze-pump-thaw cycles, the tube was sealed and heated to $120{ }^{\circ} \mathrm{C}$ for 3 days. Then the yellow-brown precipitate was collected through filtration and fully washed with $N, N$-Dimethylformamide, acetone, and tetrahydrofuran. The product was dried under a vacuum at $60{ }^{\circ} \mathrm{C}$ to obtain COF-SH.

\subsection{Fabrication of COF@Au NPs}

$200 \mathrm{mg}$ of the synthesized COF-SH was dispersed in $60 \mathrm{~mL}$ of tetrahydrofuran, followed by the addition of $\mathrm{HAuCl}_{4}$ solution $(10 \mathrm{~mL}$, $0.1 \mathrm{wt} \%)$. After stirring at room temperature for $2 \mathrm{~h}, \mathrm{NaBH}_{4}$ aqueous solution $(10 \mathrm{~mL}, 0.53 \mathrm{M})$ was rapidly added and the mixture was continued reaction overnight. The products COF@Au NPs were collected by centrifugation, fully washed with DI water and ethanol, and finally dried under a vacuum at $60{ }^{\circ} \mathrm{C}$ overnight.

\subsection{Fabrication of SMt@COF@Au NPs heterostructure}

Porous spherical montmorillonite (SMt) was prepared with the granulation of Mt. through spray-drying technology. After coated with polydopamine (PDA), the PDA-coated SMt was introduced to the COF@Au NPs suspension to obtain a mixture with a PDA-coated SMt concentration of $20 \mathrm{~g} \mathrm{~L}^{-1}$ and COF@Au NPs concentration of $1 \mathrm{~g} \mathrm{~L}^{-1}$, respectively. After being shaken for $12 \mathrm{~h}$, the product was fully washed with DI water and dried at $60{ }^{\circ} \mathrm{C}$ in a vacuum oven for at least $12 \mathrm{~h}$ to a constant weight. The content of Au NPs was determined by the ICP-MS with the loading of $0.12 \mathrm{wt} \%$. In addition, SMt@Au NPs heterostructure was fabricated through PDA chemistry for control.

\subsection{Adsorption experiment}

$20 \mathrm{mg}$ of samples were added to a $100 \mathrm{~mL}$ aqueous solution containing $40 \mathrm{mg} \cdot \mathrm{L}^{-1} \mathrm{MB}$. The whole adsorption process was monitored by the UV-Vis absorption spectra. The removal percentage $(R)$ was obtained from the Eq. (1):

$R=\left(C_{0}-C_{t}\right) / C_{0} \times 100 \%$

where $C_{0}$ and $C_{t}$ are the initial organic pollutant concentration and concentration of the reaction time, respectively.

\subsection{Evaluation of catalytic performances}

MB was selected as a model and used to assess the catalytic activity of SMt@COF@Au NPs heterostructure. The time-dependent concentration of MB was monitored by UV-Vis spectrometry. Typically, $10 \mathrm{~mL}$ of MB solution with the concentration of $250 \mathrm{mg} \mathrm{L}^{-1}$ and $10 \mathrm{~mL}$ of $\mathrm{NaBH}_{4}$ aqueous solutions with the concentration of $0.5 \mathrm{M}$ was first mixed with 80 mL DI water, then 40 mg of SMt@COF@Au NPs heterostructure was added. For recyclability, SMt@COF@Au NPs heterostructure was separated from the mixture through self-sedimentation and reused for the next cycle. The influences of the concentrations of SMt@COF@Au NPs heterostructure, reaction temperature, and $\mathrm{pH}$ values on apparent reduction rate constant $\left(K_{\mathrm{app}}\right)$ were investigated.

\section{7. $p H_{z p c}$ determination}

SMt@COF@Au NPs heterostructure was added to $10 \mathrm{~mL}$ of an aqueous solution containing $0.1 \mathrm{M} \mathrm{NaCl}$ to obtain a suspension with a SMt@COF@Au NPs heterostructure concentration of $30 \mathrm{~g} \cdot \mathrm{L}^{-1}$. The $\mathrm{pH}$ of the solution was adjusted in the range of $3-11$. Then the solution was 
$\mathbf{a}$

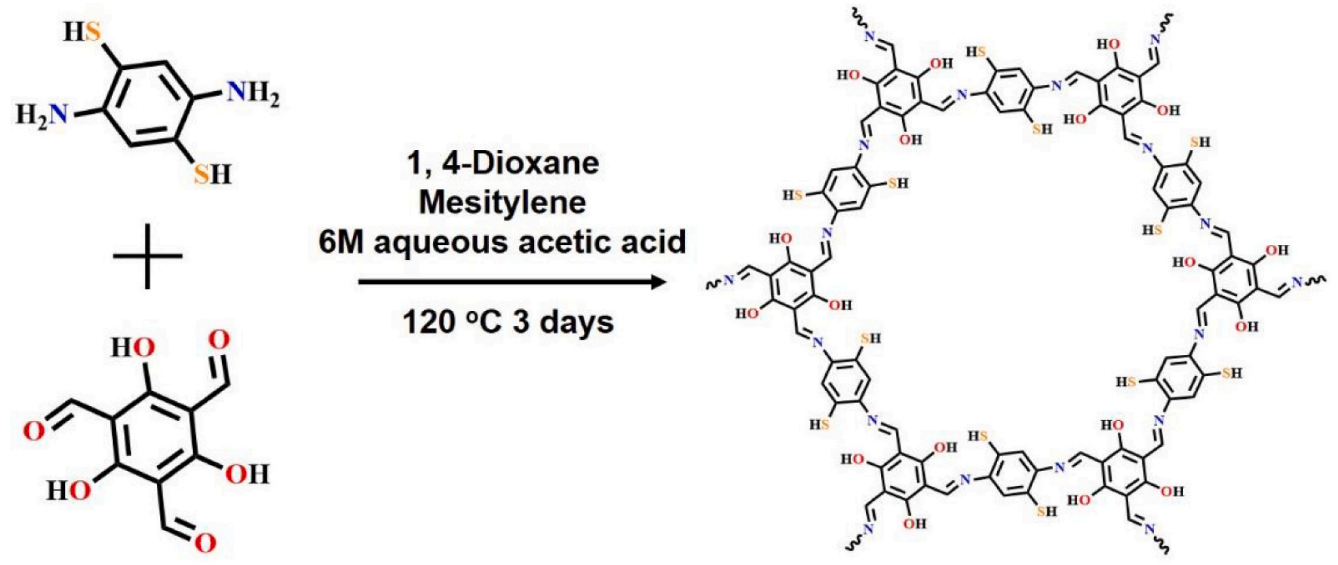

b

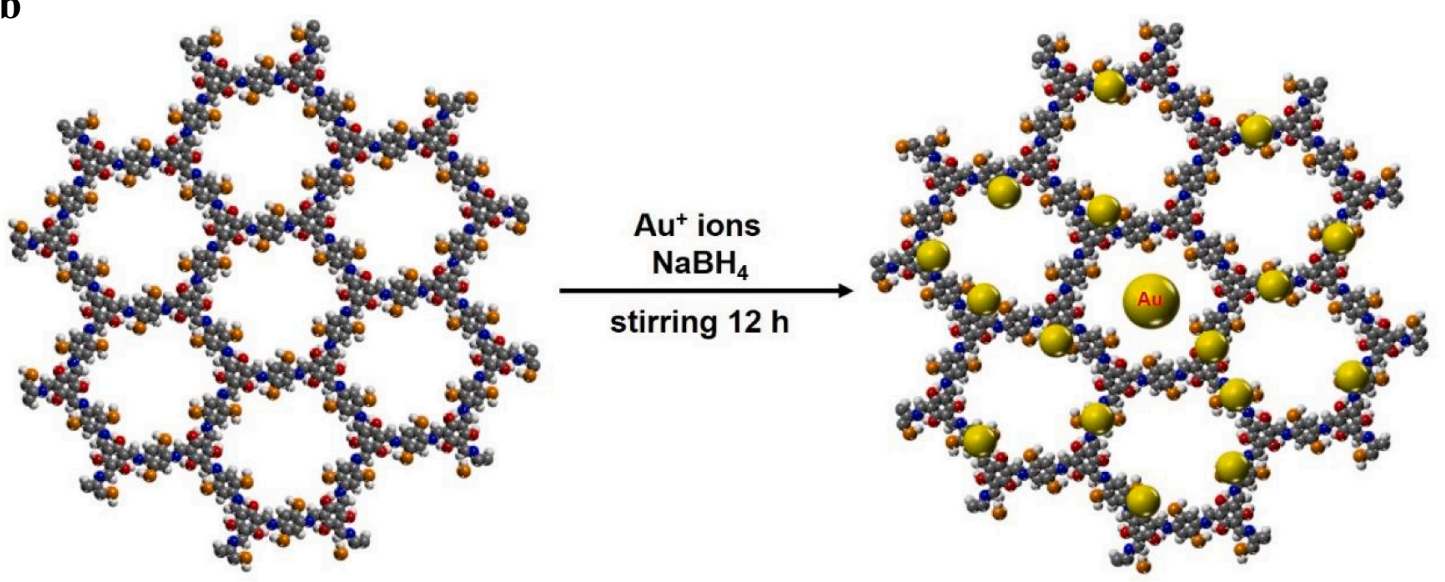

c
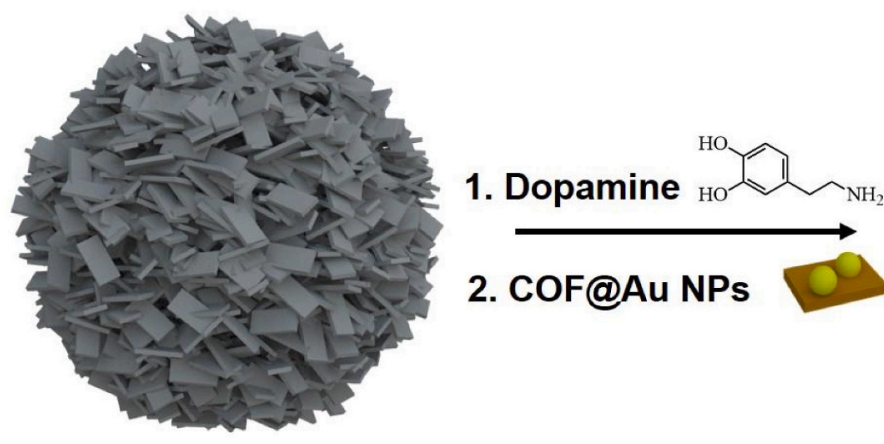

2. COF@Au NPs

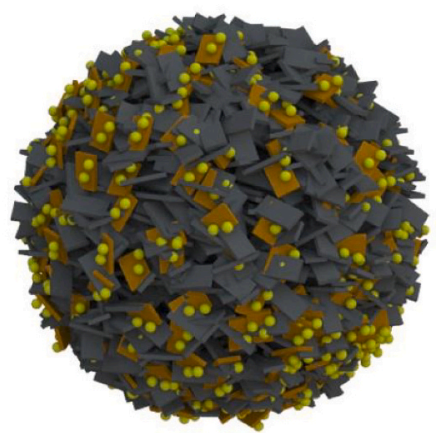

Scheme 1. The fabrication process of SMt@COF@Au NPs heterostructure including (a) the condensation reaction, (b) the chelation and reduction of Au ions on the COF substrate, and (c) the fabrication of SMt@COF@Au NPs heterostructure. The organic sheets and yellow points stand for COF and Au NPs, respectively.

fully mixed overnight and the SMt@COF@Au NPs heterostructure was picked up. The final $\mathrm{pH}$ was measured and the difference between the initial and final $\mathrm{pH}$ was plotted against the initial $\mathrm{pH}$ to obtain the $\mathrm{pH}_{\mathrm{zpc}}$.

\section{Results and discussion}

\subsection{Synthesis and characterization of the SMt@COF@Au NPs heterostructure}

COF with sulfhydryl functional groups was first synthesized through the condensation reaction with the 2, 4, 6-trihydroxybenzene-1, 3, 5-tricarbaldehyde as the knot and 2, 5-Diamino-1, 4-benzenedithiol dihydrochloride as the organic linker at high temperature. After reaction 3 days, yellow precipitate COF-SH was obtained. Then the dried COF-SH was used as a carrier to in situ supporting Au NPs to fabricate COF@Au NPs nanocomposites. On the other hand, granular SMt was surface-functionalized with polydopamine (PDA) and then used as the substrate to support COF@Au NPs nanocomposites to fabricate SMt@COF@Au NPs heterostructure, as shown in Scheme 1.

\subsection{Structural studies}

The synthesized COF was first characterized by the solid-state ${ }^{13} \mathrm{C}$ cross-polarization magic angle spinning (CP-MAS) NMR spectrum. As shown in Fig. 1a, five intense peaks between 60 and 180 ppm could be observed and the chemical shift at $164.8 \mathrm{ppm}$ indicated the condensation reaction happened between 1, 3, 5-Benzenetricarboxaldehyde and 2, 5-Diamino-1, 4-benzenedithiol dihydrochloride (Ma et al., 2019). The 
a

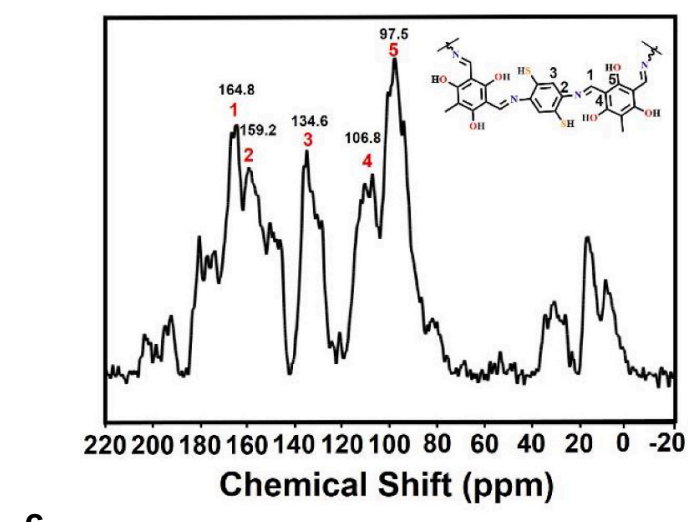

C

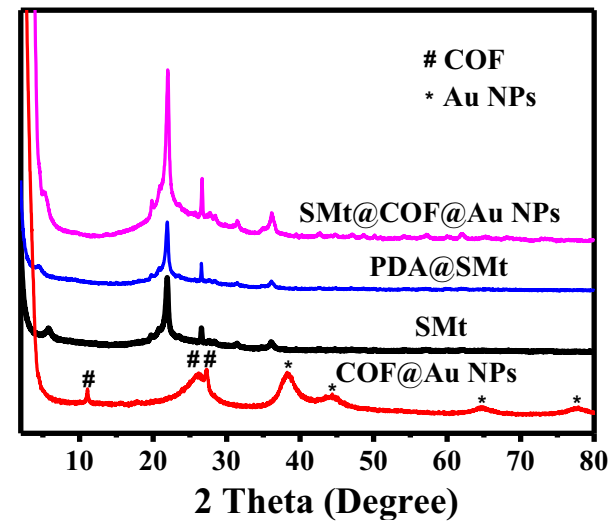

b

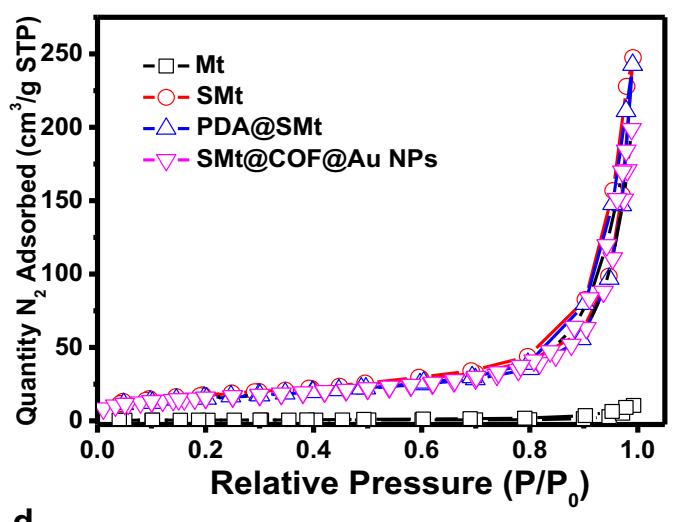

d

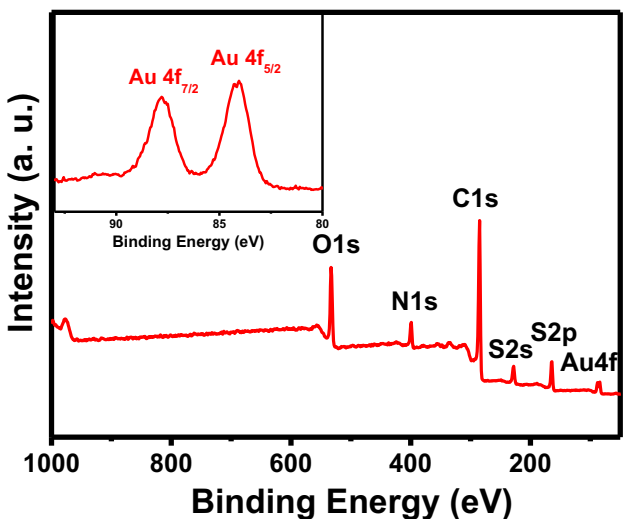

Fig. 1. (a) ${ }^{13} \mathrm{C}$ CP/MAS NMR Spectrum of COF, (b) $\mathrm{N}_{2}$ adsorption-desorption isotherms of samples, (c) XRD patterns, and (d) XPS spectrum of COF@Au NPs nanocomposites. Inset: high-resolution XPS spectrum of Au $4 \mathrm{f}$.

Table 1

Mesoscale properties of Mt., SMt, PDA@SMt, and SMt@COF@Au NPs heterostructure.

\begin{tabular}{lll}
\hline Sample & BET surface area $\left(\mathrm{m}^{2} \mathrm{~g}^{-1}\right)$ & Pore volume $\left(\mathrm{cm}^{3} \mathrm{~g}^{-1}\right)$ \\
\hline Mt & 8.1 & 0.10 \\
SMt & 61.2 & 0.38 \\
PDA@SMt & 46.4 & 0.25 \\
SMt@COF@Au NPs & 56.1 & 0.31 \\
\hline
\end{tabular}

porous structures of the prepared samples were investigated by the measurement of $\mathrm{N}_{2}$ sorption. As shown in Fig. 1b, all the samples exhibited regular pore structures typical of mesoporous materials. The pristine Mt. owned a specific surface area and pore volume of $8.1 \mathrm{~m}^{2} \mathrm{~g}^{-1}$ and $0.10 \mathrm{~cm}^{3} \mathrm{~g}^{-1}$, respectively (Table 1 ). After the granulation by the spray-drying technology, these values increased obviously to $61.2 \mathrm{~m}^{2}$ $\mathrm{g}^{-1}$ and $0.38 \mathrm{~cm}^{3} \mathrm{~g}^{-1}$, respectively (Table 1). This result reflected the efficient stacking and pore-making ability of spray drying (StundaZujeva et al., 2017). The granular SMt coated by polydopamine (PDA) decreased these values to $46.4 \mathrm{~m}^{2} \mathrm{~g}^{-1}$ and $0.25 \mathrm{~cm}^{3} \mathrm{~g}^{-1}$, respectively, suggesting the weak $\mathrm{N}_{2}$ adsorption ability of PDA. Conversely, these values increased to $56.1 \mathrm{~m}^{2} \mathrm{~g}^{-1}$ and $0.31 \mathrm{~cm}^{3} \mathrm{~g}^{-1}$, respectively, after supporting COF@Au NPs, demonstrating the efficient $\mathrm{N}_{2}$ adsorption ability of COF (Table 1). Furthermore, XRD was used to confirm the crystallinity of the prepared COF and the loading of Au NPs on the SMt@COF@Au NPs heterostructure. As shown in Fig. 1c, three obvious peaks at $2 \theta$ of $11.0^{\circ}, 26.3^{\circ}$, and $27.4^{\circ}$ could be observed from the XRD curve of COF/Au NPs, resulting from the appearance of long-range order structure (Deng et al., 2020). Four strong peaks at $2 \theta$ of $38.1^{\circ}, 44.4^{\circ}$, $64.5^{\circ}$, and $77.8^{\circ}$ can be observed, which is well following the JCPDS card no. 04-784, demonstrating the successful supporting of Au NPs on the COF surface (Xiao et al., 2019). Compared with SMt, the SMt@COF@Au NPs patterns exhibited similar features (JCPDS card no. 13-0135 for smectite and JCPDS card no. 46-1045 for quartz, respectively) and the same crystallite size $(14.5 \mathrm{~nm})$, indicating the higher content of SMt in the SMt@COF@Au NPs heterostructure (Gonçalves et al., 2017). XPS was further carried out to provide information on the surface elemental composition of the samples. As shown in Fig. 1d, the peaks corresponding to $\mathrm{C}, \mathrm{O}, \mathrm{N}, \mathrm{S}$, and Au could be observed, well consistent with the formation of COF@Au NPs nanocomposites. From the inset of Fig. 1d, the binding energies at $86.6 \mathrm{eV}$ and $83.0 \mathrm{eV}$ could be attributed to $A u 4 f_{7 / 2}$ and $A u 4 f_{5 / 2}$ of $A u(0)$, respectively, confirming the loading of $\mathrm{Au}(0) \mathrm{NPs}$ on the surface of COF. As shown in Fig. S1, the peaks corresponding to $\mathrm{C}, \mathrm{O}, \mathrm{N}, \mathrm{Si}$, and $\mathrm{S}$ could be observed, demonstrating the well supporting of COF@Au NPs nanocomposites on SMt. However, no Au peak could be observed, reflecting the lower content of $\mathrm{Au}$ on the SMt@COF@Au NPs heterostructure. ICP/MS was used to measure the loading of Au NPs. The content of Au NPs was quantified to be 0.12 wt $\%$ after dissolving the SMt@COF@Au NPs heterostructure in the strong acid. Besides, Au NPs were quantified to be $3.92 \mathrm{wt} \%$ in the COF@Au NPs nanocomposites. Thus, the loading of COF@Au NPs nanocomposites on the SMt@COF@Au NPs heterostructure could be calculated as $2.94 \mathrm{wt} \%$.

The production of a catalyst with controllable morphology can not only improve the regularity of products and flowability but also decreases fouling, facilitating mass production for application (Qin et al., 2011). Therefore, SEM was used here to observe the morphologies of the fabricated samples. A granular morphology with a size distribution (17 $\pm 4 \mathrm{~nm}$ ) was observed from Fig. 2a and Fig. S2. The synthesized COF exhibited a multilayer lamellar structure with a smooth surface 

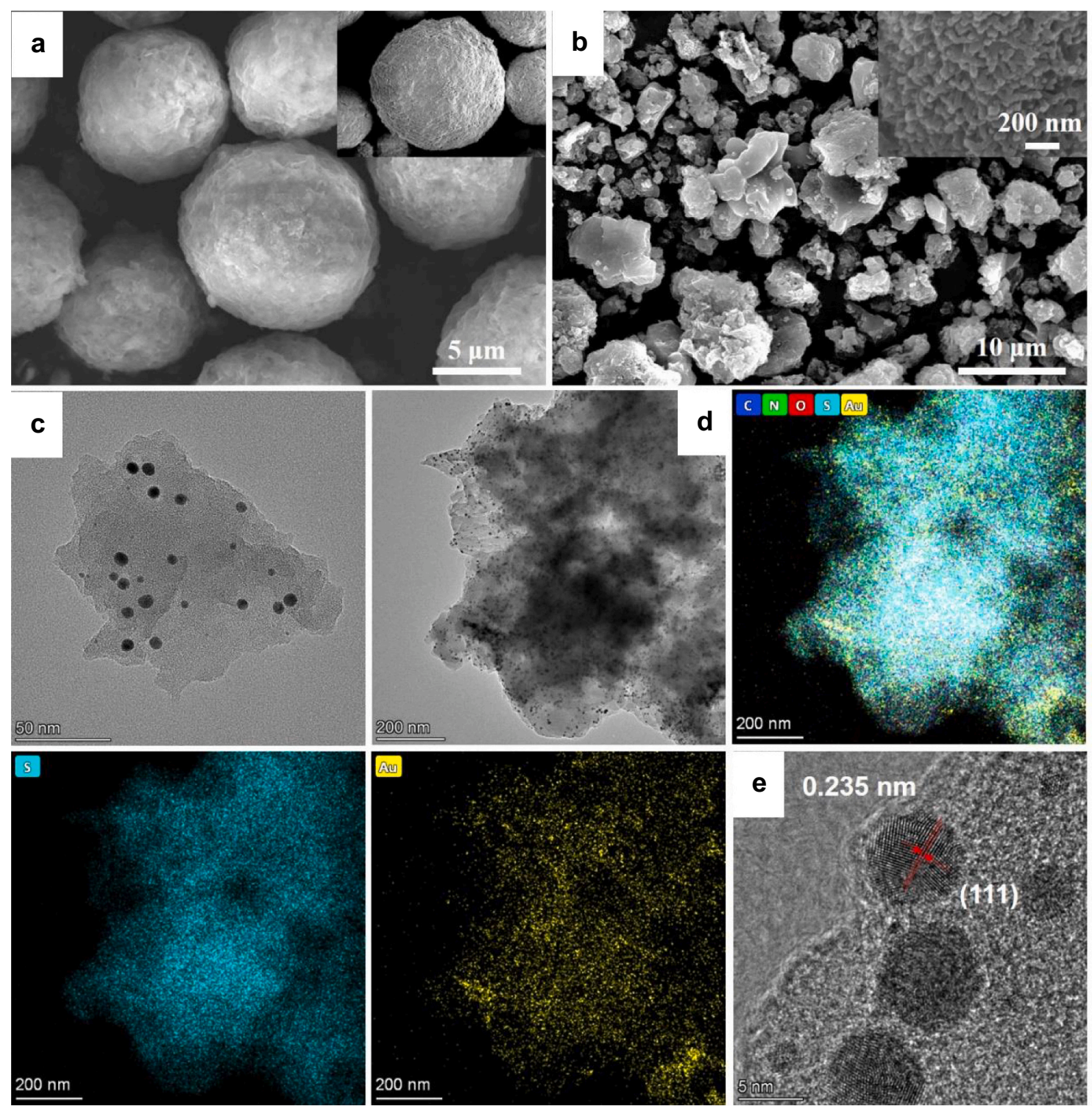

Fig. 2. SEM images of the (a) SMt@COF@Au NPs heterostructure with inset of pristine SMt and (b) COF with inset enlarging images, (c) TEM image of COF@Au NPs dispersed in aqueous solution, (d) TEM-EDX elemental mapping images, and (e) high solution TEM image of the SMt@COF@Au NPs heterostructure.

(Fig. 2b). From the inset of Fig. 2b, the nanowires with an average diameter of about $40 \mathrm{~nm}$ could be observed. The morphology of COF@Au NPs nanocomposites after dissolving in an aqueous solution was observed by TEM. As shown in Fig. 2c, tiny Au NPs with a narrow size distribution $(4 \pm 1 \mathrm{~nm}$ ) were uniformly dispersed on the surface of COF. The size of dispersed COF was about $50 \mathrm{~nm}$, which is much suitable for supporting by micro-sized substrate SMt. Compared with SMt (inset of Fig. 2a), a smooth and lamellar structure could be seen on the surface of SMt@COF@Au NPs heterostructure, suggesting the good wrapping of COF@Au NPs nanocomposites on the surface of SMt. The uniformly wrapping of COF@Au NPs on the surface of SMt is mainly due to the excellent adhesion ability of polydopamine coating. Corresponding to the TEM image, the EDX mapping of $\mathrm{C}, \mathrm{N}, \mathrm{O}, \mathrm{S}$, and Au elements exhibited in the same regions, demonstrating the fixed supporting of COF@Au NPs nanocomposites in the synthesized heterostructure (Fig. 2d). HR-TEM was employed to further prove the successful supporting of Au NPs on the SMt@COF@Au NPs heterostructure. As shown in Fig. 2f, Au NPs with the inter-planar spacing of $0.235 \mathrm{~nm}$ can be observed, which was assigning to the (111) plane of Au crystal (Xu et al., 2020). Although the content of Au NPs is lower in the SMt@COF@Au
NPs heterostructure, the small tiny size and uniform dispersion of Au NPs could supply the abundant active sites for catalytic reduction of organic pollutants.

\subsection{Catalytic properties of SMt@COF@Au NPs heterostructure}

Ultrafine noble metal nanoparticles with a diameter of less than $5 \mathrm{~nm}$ exhibited ultrahigh catalytic activities towards organic pollutants due to their large surface area to provide enough activity sites for electron transfer (Gong et al., 2019). Hence, the catalytic activity of the synthesized samples was evaluated by the reduction of carcinogenic toxic organic pollutant methylene blue (MB) in the presence of reduced agent $\mathrm{NaBH}_{4}$ (Jana et al., 2019; Joseph et al., 2019; Lackovičová et al., 2019). The reduction process was monitored by a time-resolved UV-Vis spectroscopy. As shown in Fig. 3a, the peak at $665 \mathrm{~nm}$ gradually vanished within $3 \mathrm{~min}$, reflecting the conversion of carcinogenic $\mathrm{MB}$ to inactive leuco-methylene blue. The sample prepared without COF was taken for control. As shown in Fig. S3, SMt@Au NPs with Au NPs with an average diameter size of $80 \mathrm{~nm}$ and content of $4 \mathrm{wt} \%$ were synthesized under similar conditions. More than $8 \mathrm{~min}$ was spent finishing the reaction 
a

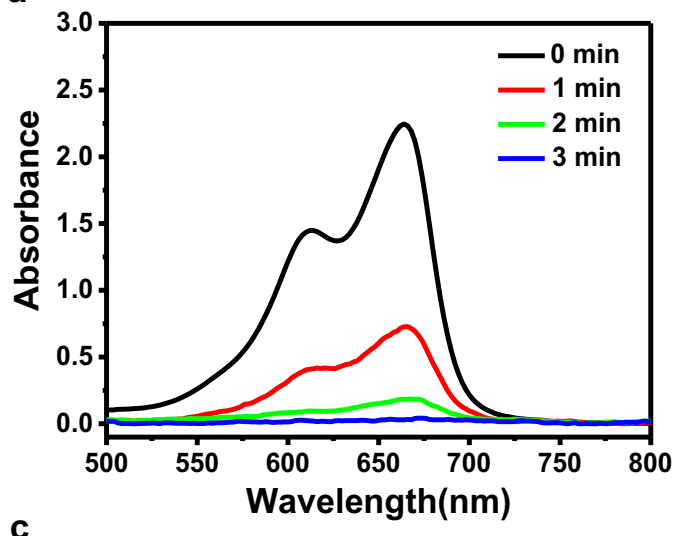

C

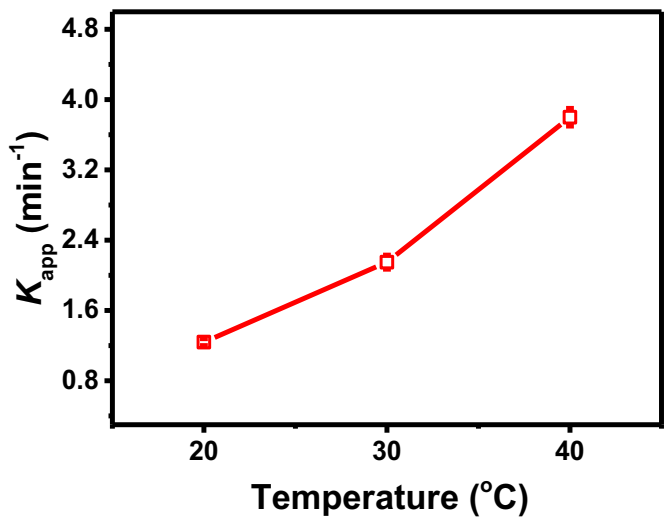

e

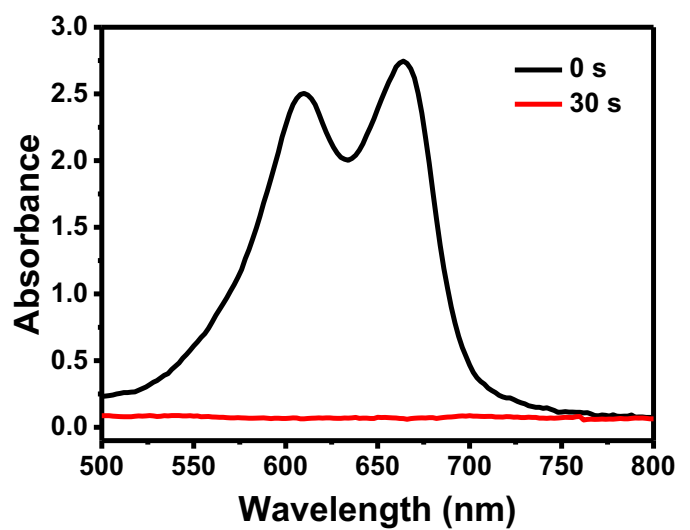

b
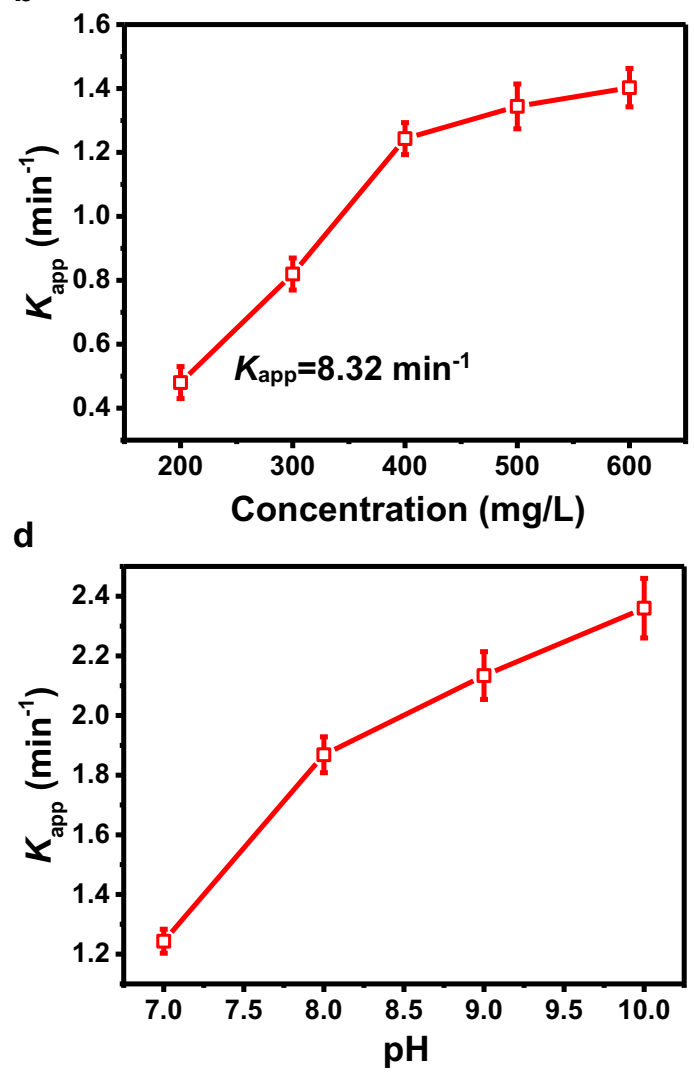

f

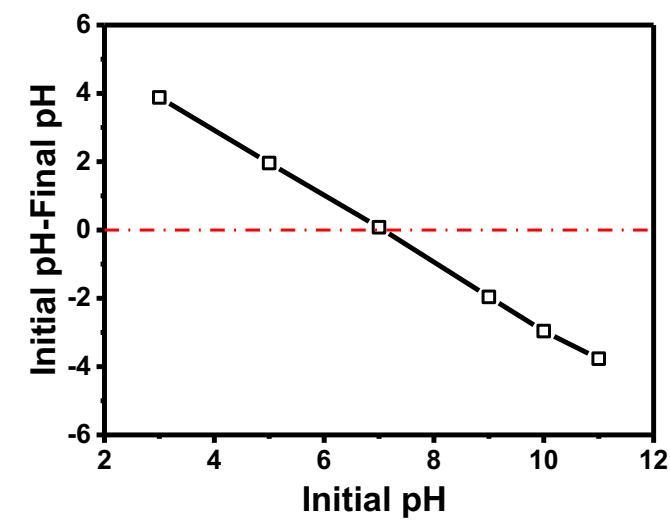

Fig. 3. (a) The time-resolved UV-vis spectra of MB. The effect of (b) SMt@COF@Au NPs heterostructure concentration, (c) temperature, and (d) pH values on the catalytic performance. (e) The time-resolved UV-Vis spectra of MB, the reaction conditions: $25 \mathrm{mg} \mathrm{L}^{-1} \mathrm{MB}, 400 \mathrm{mg} \mathrm{L}{ }^{-1}$ SMt@COF@Au NPs, pH 10, and temperature $40{ }^{\circ} \mathrm{C}$. (f) $\mathrm{pH}_{\mathrm{zpc}}$ determination of SMt@COF@Au NPs heterostructure.

Table 2

The comparison of catalytic activities of noble metal-based heterogenous catalysts reported recently for MB reduction with $\mathrm{NaBH}_{4}$.

\begin{tabular}{lllll}
\hline Catalyst & BET surface area $\left(\mathrm{m}^{2} \mathrm{~g}^{-1}\right)$ & $K_{\text {app }}\left(\mathrm{min}^{-1}\right)$ & TOF $\left(\mathrm{h}^{-1}\right)$ & Ref. \\
\hline $\mathrm{Fe}_{3} \mathrm{O}_{4} @$ COF-Au & 193.4 & 0.90 & $6.4 \times 10^{4}$ & Appl. Catal. B Environ., 2020 (Xu et al., 2020) \\
$\mathrm{SMt} @ \mathrm{Ag}$ & 45.0 & 0.24 & 110 & App. Clay Sci., 2019 (Wang et al., 2019) \\
$\mathrm{MgAlCe-LDH@Au} \mathrm{NPs}$ & - & 1.80 & 2200 & J. Mater. Chem. A, 2017 (Iqbal et al., 2017) \\
$\mathrm{MGO-TA@Fe}{ }^{3+} @ \mathrm{Ag}$ & 126.8 & 3.24 & 6404 & Chem. Eng. J., 2021 (Yang et al., 2021) \\
Graphene@Ag NPs & - & 0.25 & 2120 & ACS Sustain. Chem. Eng., 2018 (Sahoo et al., 2018) \\
$\mathrm{Fe}_{3} \mathrm{O}_{4} @ E D T A @ A g$ & 1.83 & 198 & ACS Appl. Nano Mater. 2019 (Sharif et al., 2019) \\
SMt@COF@Au NPs & 81.0 & 8.24 & $3.2 \times 10^{5}$ & this work \\
\hline
\end{tabular}

(Fig. S4). Considered the higher Au NPs, the rate was much less than our prepared SMt@COF@Au NPs heterostructure. These results demonstrated the good size control of COF towards the growth of Au NPs and the excellent electron transfer ability of COF@Au NPs nanocomposites towards MB. Furthermore, the influences of the concentration of the catalyst, reaction temperature, and $\mathrm{pH}$ value were investigated. 

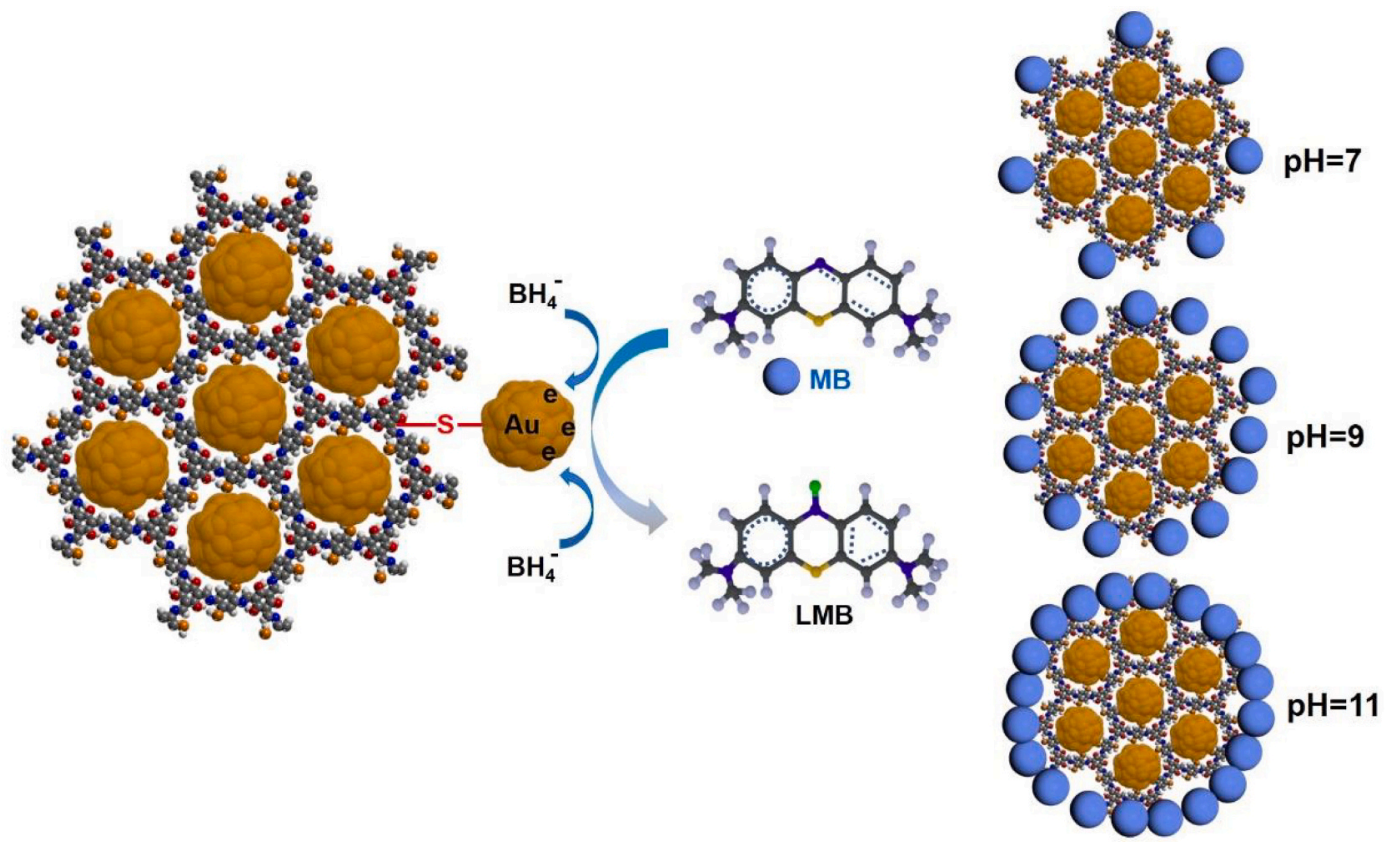

Scheme 2. Mechanism of the catalytic reduction of MB with SMt@COF@Au NPs heterostructure.

Considering the excess concentration of $\mathrm{NaBH}_{4}$ existing in the catalytic system, the kinetic equation $\ln \left(C_{\mathrm{o}} / C_{t}\right)=K_{\text {app }} t$ was used to evaluate the catalytic performance, where the initial concentration of $\mathrm{MB}\left(C_{0}\right)$ and the concentration of $\mathrm{MB}$ at the time $t\left(C_{t}\right)$ were calculated from the calibration curves (Fig. S5), where $K_{\text {app }}$ is the apparent first-order rate constant (Zhang et al., 2014). As shown in Fig. 3b, $K_{\text {app }}$ increased from $0.48 \mathrm{~min}^{-1}$ to $1.24 \mathrm{~min}^{-1}$ with the concentration of SMt@COF@Au NPs heterostructure increasing from $200 \mathrm{mg} \mathrm{L}^{-1}$ to $400 \mathrm{mg} \mathrm{L}^{-1}$. $K_{\text {app }}$ reached a plateau with further increasing the concentration of SMt@COF@Au NPs heterostructure from $400 \mathrm{mg} \mathrm{L}^{-1}$ to $600 \mathrm{mg} \mathrm{L}^{-1}$, reflecting enough a
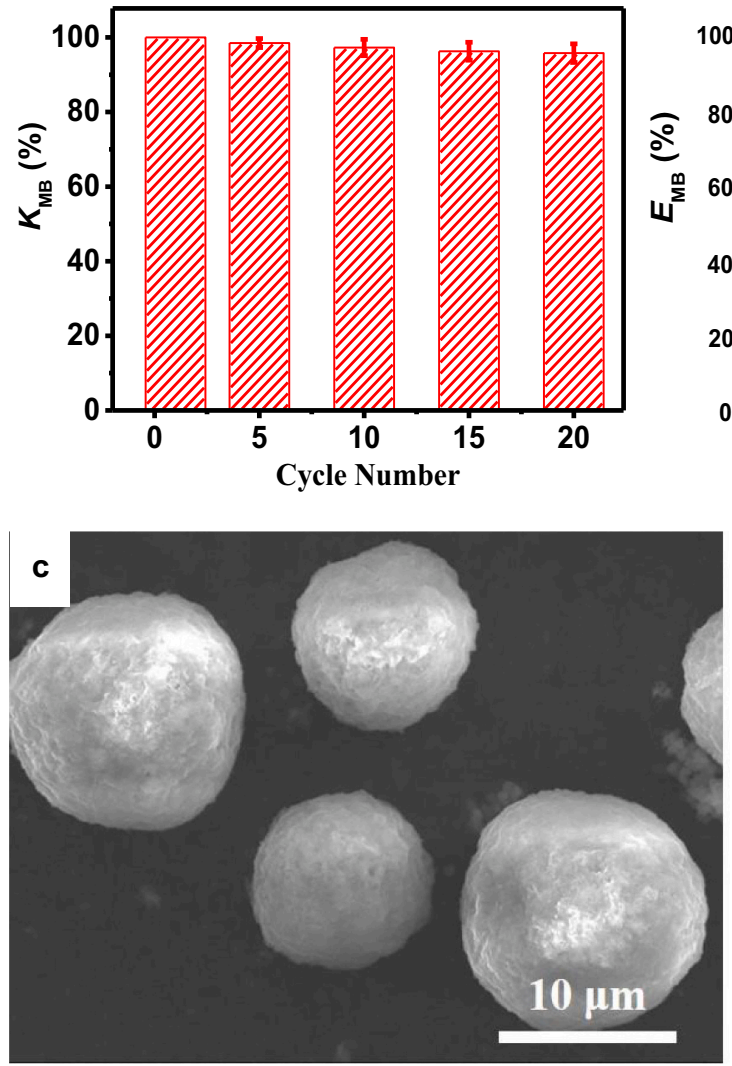

b

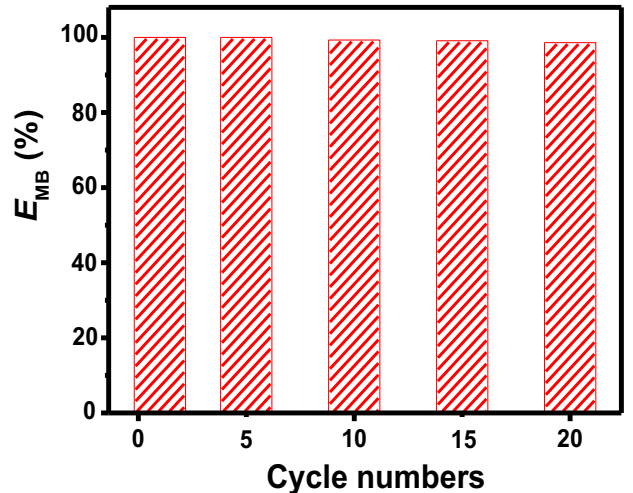

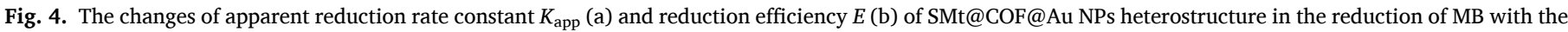
prolonged cycle number. (c) SEM and (d) TEM images of SMt@COF@Au NPs heterostructure after cycled 20 times. 


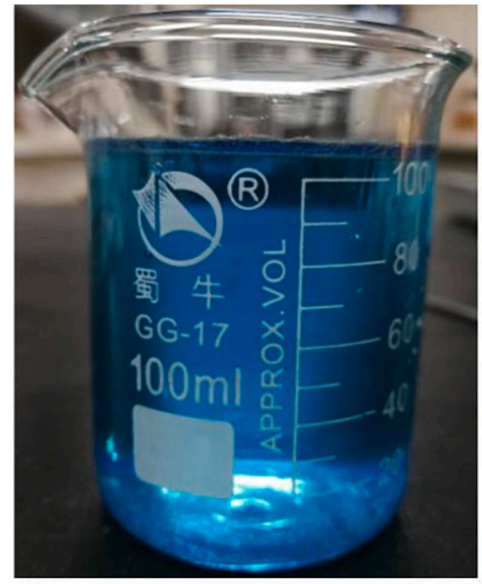

Seawater/MB

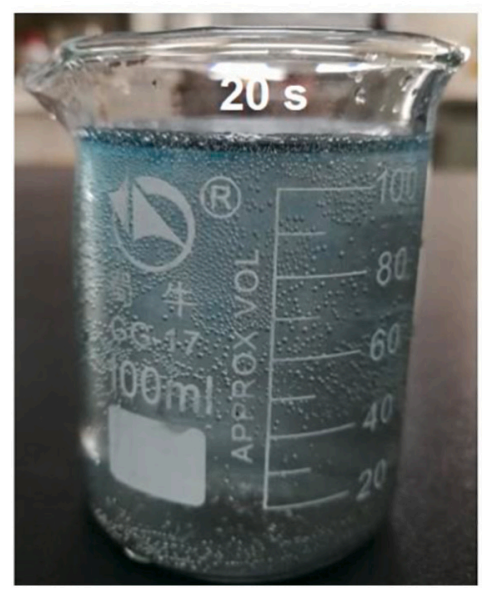

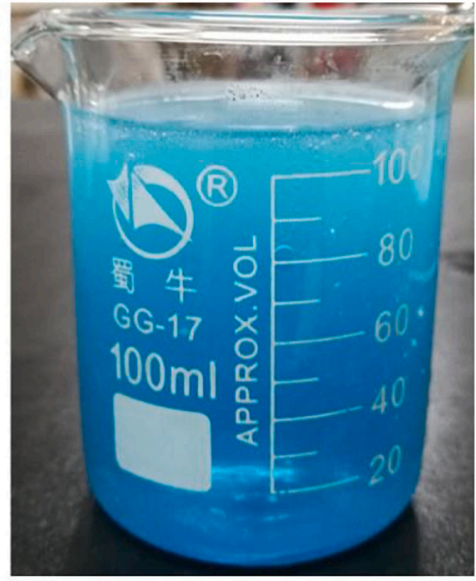

Seawater/MB/NaBH

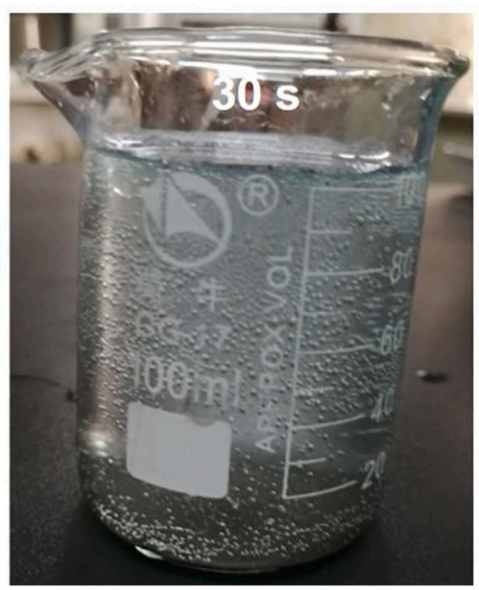

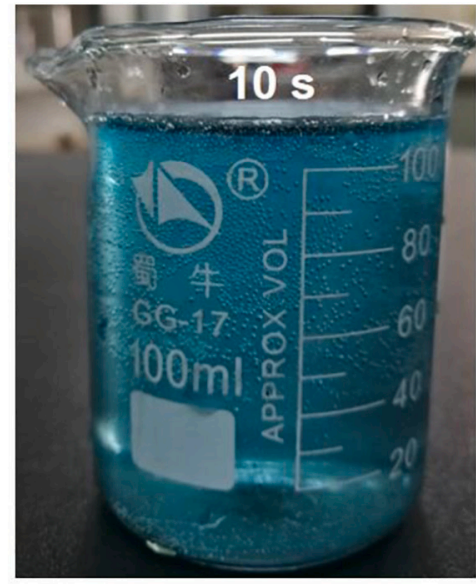

Seawater/MB/NaBH ${ }_{4} /$ SMt@COF@Au NPs

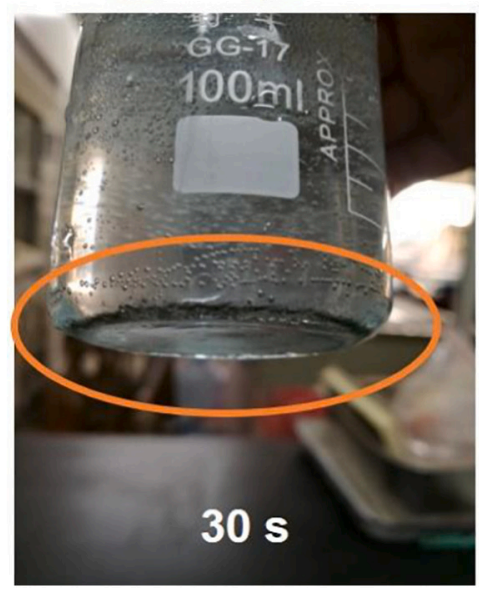

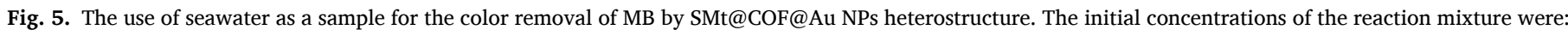
MB (25 mg L $\left.{ }^{-1}\right), \mathrm{NaBH}_{4}(0.05 \mathrm{M})$, and SMt@COF@Au NPs heterostructure (1000 mg L $\left.{ }^{-1}\right)$.

active sites providing by SMt@COF@Au NPs heterostructure for electron transfer. The value of $K_{\text {app }}$ increased from $1.24 \mathrm{~min}^{-1}$ to $3.80 \mathrm{~min}^{-1}$ with the reaction temperature increasing from $20^{\circ} \mathrm{C}$ to $40^{\circ} \mathrm{C}$ (Fig. 3c). The value of $K_{\text {app }}$ increased from $1.24 \mathrm{~min}^{-1}$ to $2.36 \mathrm{~min}^{-1}$ with the increase of the $\mathrm{pH}$ values from 7 to 10 . Hence, the optimal $K_{\text {app }}(8.32$ $\mathrm{min}^{-1}$, Fig. 3e) in our experiment was obtained at the condition of 400 $\mathrm{mg} \mathrm{L}^{-1} \mathrm{SMt} @ \mathrm{COF} @ A u$ NPs heterostructure and temperature $40^{\circ} \mathrm{C}$ and $\mathrm{pH}=10$. The reaction was finished within $30 \mathrm{~s}$. Moreover, the turnover frequency (TOF) of SMt@COF@Au NPs heterostructure was calculated to be $3.2 \times 10^{5} \mathrm{~h}^{-1}$, demonstrating the excellent catalytic activity towards organic pollutant MB compared with those of noble metal-based catalysts reported recently (Table 2).

\subsection{The catalytic reduction mechanism}

The mechanism based on the high catalytic activity of SMt@COF@Au NPs heterostructure towards MB was proposed here. The main reason could be attributed to the special structure of the prepared heterostructure. Firstly, COF functionalized with sulfhydryl groups was designed and synthesized, by which the size of Au NPs was limited and $\mathrm{Au}$ NPs were immobilized on the surface of COF (Zhang et al., 2020c). The tiny small size Au NPs could provide abundant active sites for fast electron transfer. Secondly, COF was a good semiconductor, could serve as a cocatalyst to strengthen the electron transfer of Au NPs (Wang et al., 2021; Zhang et al., 2020a). Thirdly, the adsorption ability of
SMt@COF@Au NPs heterostructure could enhance the concentration of MB nearby the Au NPs, thus accelerating the catalytic reduction rate (Xiao et al., 2019). Furthermore, the influence of $\mathrm{pH}$ on the catalytic activities of SMt@COF@Au NPs heterostructure was investigated. As shown in Fig. S6, the $\mathrm{R}_{\mathrm{MB}}$ increased from $13.0 \%$ to $25.4 \%$ as the increase of $\mathrm{pH}$ values from 4 to 11 . Hence, $\mathrm{pHzpc}$ was detected to prove the relationship between the adsorption and $\mathrm{pH}$ values (Zhang et al., 2020b). As shown in Fig. 3f, the value of $\mathrm{pH}_{\mathrm{zpc}}$ was 7.1, that is, when the pH values exceeded 7.1, the surface of the SMt@COF@Au NPs heterostructure was negatively charged. For $\mathrm{MB}$, a cationic organic pollutant, the electrostatic adsorption with the SMt@COF@Au NPs heterostructure would strengthen with the increase of $\mathrm{pH}$ values (Fig. S7). Therefore, a higher adsorption value was obtained at high $\mathrm{pH}$ values. The enhanced adsorption ability at high $\mathrm{pH}$ value could increase the concentration of organic pollutants around the active sites, thus accelerating the catalytic reaction (Scheme 2).

\subsection{The stability of SMt@COF@Au NPs heterostructure}

The recovery and reuse of noble metal-based catalysts are significantly important for the treatment of wastewater containing organic pollutants. Hence, successive 20 cycles were carried out to evaluate the stability of the SMt@COF@Au NPs heterostructure. As shown in Fig.4a and Fig. $4 \mathrm{~b}$, the values of $K_{\text {app }}$ slightly decreased $2 \%$ and $R_{\mathrm{MB}}$ maintained unaltered after 20 cycles. Besides, from the SEM images (Fig. 4c) and 
TEM images (Fig. 4d), the morphology was unchanged after 20 cycles. The excellent stability of the prepared SMt@COF@Au NPs heterostructure can be attributed to the strong interfacial interaction between SMt and COF@Au NPs through the adhesion of polydopamine as well as the strong interfacial interaction between COF and Au NPs through the S-Au bonding (Zhang et al., 2020c).

\subsection{Practical application}

Seawater collecting from the Yellow Sea, Yantai, Shandong Province, China, was used here for practical application. As shown in Fig. 5, after adding $\mathrm{NaBH}_{4}$ and SMt@COF@Au NPs heterostructure to the seawater/ MB solution, the color vanished quickly within 30 s. SMt@COF@Au NPs heterostructure can quickly deposit on the bottom and can be easily reused without any devices involved. Therefore, the application of SMt@COF@Au NPs heterostructure for practical wastewater treatment is highly desirable.

\section{Conclusions}

A new covalent organic framework (COF) with sulfhydryl groups was designed to provide basic sites for Au ions, by which small size gold nanoparticles (Au NPs) were immobilized on the COF to form COF@Au NPs nanocomposites. The prepared nanocomposites were further stabilized supported by spherical montmorillonite (SMt) through the polydopamine chemistry method. The resultant heterostructure exhibited a high apparent reduction rate constant value $\left(8.32 \mathrm{~min}^{-1}\right)$ and TOF (3.2 $\times 10^{5} \mathrm{~h}^{-1}$ ) towards organic pollutant methylene blue (MB). The high catalytic activity of our prepared heterostructure can be contributed to the strong adsorption ability of SMt towards MB, which increased the concentration of MB near COF@Au NPs, accelerating the catalytic reaction. Furthermore, the prepared heterostructure can be facile recovery due to the self-sedimentary ability of SMt. Moreover, the catalytic activity could maintain almost unalter after a successive cycle for 20 times. This work demonstrated the design and synthesis of a new granular SMt@COF@Au NPs heterostructure for mass production and catalytic application through the integration of COF@Au NPs and SMt. We believed that our strategy would pave a new way for the application of noble-metal based heterogeneous catalyst for treating wastewater containing organic pollutants.

\section{Author contributions}

All authors have given approval to the final version of the manuscript.

\section{Declaration of Competing Interest}

The authors declare that they have no known competing financial interests or personal relationships that could have appeared to influence the work reported in this paper.

\section{Acknowledgments}

The authors gratefully acknowledge the funding support from National Natural Science Foundation of China (No. 51903247), Key Science and Technology Program of Yantai City (No. 2018ZHGY075 and 2019XDHZ093), and Shandong Key Laboratory of Coastal Environmental Processes, YICCAS (Grant No. 2019SDHADKFJJ15).

\section{Appendix A. Supplementary data}

Supplementary data to this article can be found online at https://doi. org/10.1016/j.clay.2021.106007.

\section{References}

Arora, N., Mehta, A., Mishra, A., Basu, S., 2018. 4-Nitrophenol reduction catalysed by $\mathrm{Au}-\mathrm{Ag}$ bimetallic nanoparticles supported on LDH: homogeneous vs. heterogeneous catalysis. Appl. Clay Sci. 151, 1-9.

Cao, H.L., Huang, H.B., Chen, Z., Karadeniz, B., Lü, J., Cao, R., 2017. Ultrafine silver nanoparticles supported on a conjugated microporous polymer as highperformance nanocatalysts for nitrophenol reduction. ACS Appl. Mater. Interfaces 9, 5231-5236.

Cao, H.L., Liu, C., Cai, F.Y., Qiao, X.X., Dichiara, A.B., Tian, C., Lü, J., 2020. In situ immobilization of ultra-fine Ag NPs onto magnetic Ag@RF@ $\mathrm{Fe}_{3} \mathrm{O}_{4}$ core-satellite nanocomposites for the rapid catalytic reduction of nitrophenols. Water Res. 179, 115882.

Das, R., Sypu, V.S., Paumo, H.K., Bhaumik, M., Maharaj, V., Maity, A., 2019. Silver decorated magnetic nanocomposite $\left(\mathrm{Fe}_{3} \mathrm{O}_{4} @ \mathrm{PPy}-\mathrm{MAA} / \mathrm{Ag}\right)$ as highly active catalyst towards reduction of 4-nitrophenol and toxic organic dyes. Appl. Catal. B 244, $546-558$.

Deng, Y., Zhang, Z., Du, P., Ning, X., Wang, Y., Zhang, D., Liu, J., Zhang, S., Lu, X., 2020. Embedding ultrasmall au clusters into the pores of a covalent organic framework for enhanced photostability and photocatalytic performance. Angew. Chem. Int. Ed. 59, 6082-6089.

Gao, C., Lyu, F., Yin, Y., 2020. Encapsulated metal nanoparticles for catalysis. Chem. Rev. https://doi.org/10.1021/acs.chemrev.0c00237.

Gao, G., Xi, Q., Zhang, Y., Jin, M., Zhao, Y., Wu, C., Zhou, H., Guo, P., Xu, J., 2019. Atomic-scale engineering of MOF array confined Au nanoclusters for enhanced heterogeneous catalysis. Nanoscale. 11, 1169-1176.

Gonçalves, W.P., Silva, V.J., Menezes, R.R., Neves, G.A., Lira, H.L., Santana, L.N.L., 2017. Microstructural, physical and mechanical behavior of pastes containing clays and alumina waste. Appl. Clay Sci. 137, 259-265.

Gong, W., Wu, Q., Jiang, G., Li, G., 2019. Ultrafine silver nanoparticles supported on a covalent carbazole framework as high-efficiency nanocatalysts for nitrophenol reduction. J. Mater. Chem. A 7, 13449-13454.

Hou, C., Zhao, D.Y., Chen, W.Q., Li, H., Zhang, S.F., Liang, C., 2020. Covalent organic framework-functionalized magnetic $\mathrm{CuFe}_{2} \mathrm{O}_{4} / \mathrm{Ag}$ nanoparticles for the reduction of 4-nitrophenol. Nanomaterials 10, 426.

Iqbal, K., Iqbal, A., Kirillov, A.M., Wang, B., Liu, W.S., Tang, Y., 2017. New ce-doped MGAL-LDH@Au nanocatalyst for highly efficient reductive degradation of organic contaminants. J. Mater. Chem. A 5, 6716-6724.

Iqbal, K., Iqbal, A., Kirillov, A.M., Shan, C., Liu, W., Tang, Y., 2018. A new multicomponent CDs/Ag@Mg-Al-Ce-LDH nanocatalyst for highly efficient degradation of organic water pollutants. J. Mater. Chem. A 6, 4515-4524.

Jana, S., Ray, J., Mondal, B., Tripathy, T., 2019. Efficient and selective removal of cationic organic dyes from their aqueous solutions by a nanocomposite hydrogel, katira gum-cl-poly(acrylic acid-co-N, N-dimethylacrylamide)@bentonite. Appl. Clay Sci. 173, 46-64.

Joseph, A., Vellayan, K., González, B., Vicente, M.A., Gil, A., 2019. Effective degradation of methylene blue in aqueous solution using Pd-supported Cu-doped Ti-pillared montmorillonite catalyst. Appl. Clay Sci. 168, 7-10.

Lackovičová, M., Baranyaiová, T., Bujdák, J., 2019. The chemical stabilization of methylene blue in colloidal dispersions of smectites. Appl. Clay Sci. 181, 105222.

Liao, G., Fang, J., Li, Q., Li, S., Xu, Z., Fang, B., 2019. Ag-based nanocomposites: synthesis and applications in catalysis. Nanoscale. 11, 7062-7096.

Ma, W., Zheng, Q., He, Y., Li, G., Guo, W., Lin, Z., Zhang, L., 2019. Size-controllable synthesis of uniform spherical covalent organic frameworks at room temperature for highly efficient and selective enrichment of hydrophobic peptides. J. Am. Chem. Soc. $141,18271-18277$.

Peng, K., Wang, J., Wang, H., Li, X., Wan, P., Zhang, H., Bai, L., 2019. MoS 2 nanosheets supported on carbon hybridized montmorillonite as an efficient heterogeneous catalyst in aqueous phase. Appl. Clay Sci. 183, 105346.

Qin, L., Huang, D., Xu, P., Zeng, G., Lai, C., Fu, Y., Yi, H., Li, B., Zhang, C., Cheng, M., Zhou, C., Wen, X., 2019a. In-situ deposition of gold nanoparticles onto polydopamine-decorated $g_{-} \mathrm{C}_{3} \mathrm{~N}_{4}$ for highly efficient reduction of nitroaromatics in environmental water purification. J.Colloid. Interf. Sci. 534, 357-369.

Qin, L., Yi, H., Zeng, G.M., Lai, C., Huang, D.L., Xu, P.A., Fu, Y.K., He, J.F., Li, B.S., Zhang, C., Cheng, M., Wang, H., Liu, X.G., 2019b. Hierarchical porous carbon material restricted Au catalyst for highly catalytic reduction of nitroaromatics. J. Hazard. Mater. 380, 120864.

Qin, Y., Wang, N., Zhou, Y., Huang, Y., Niu, H., Dong, J.Y., 2011. Fabrication of nanofillers into a granular "nanosupport" for ziegler-natta catalysts: Towards scalable in situ preparation of polyolefin nanocomposites. Macromol. Rapid Commun. 32, 1052-1059.

Sahoo, P.K., Kumar, N., Thiyagarajan, S., Thakur, D., Panda, H.S., 2018. Freeze-casting of multifunctional cellular 3D-graphene/Ag nanocomposites: Synergistically affect supercapacitor, catalytic and antibacterial properties. ACS Sustain. Chem. Eng. 6, 7475-7487.

Sharif, H.M.A., Mahmood, A., Cheng, H.Y., Djellabi, R., Ali, J., Jiang, W.L., Wang, S.-S., Haider, M.R., Mahmood, N., Wang, A.-J., 2019. $\mathrm{Fe}_{3} \mathrm{O}_{4}$ nanoparticles coated with EDTA and Ag nanoparticles for the catalytic reduction of organic dyes from wastewater. ACS Appl. Nano. Mater. 2, 5310-5319.

Stunda-Zujeva, A., Irbe, Z., Berzina-Cimdina, L., 2017. Controlling the morphology of ceramic and composite powders obtained via spray drying-a review. Ceram. Int. 43, 11543-11551.

Wang, H., Wang, N., Wang, F., Xiao, F., Pan, D., 2020. Spherical montmorillonitesupported molybdenum disulfide nanosheets as a self-sedimentary catalyst for organic pollutants removal. Sep. Purif. Technol. 117346. 
Wang, J., Zhuang, S., 2019. Covalent organic frameworks (COFs) for environmental applications. Coord. Chem. Rev. 400, 213046.

Wang, N., Xiao, F., Zhang, J., Zhou, H., Qin, Y., Pan, D., 2019. Spherical montmorillonite-supported nano-silver as a self-sedimentary catalyst for methylene blue removal. Appl. Clay Sci. 174, 146-151.

Wang, N., Wang, F., Pan, F., Yu, S., Pan, D., 2021. Highly efficient silver catalyst supported by a spherical covalent organic framework for the continuous reduction of 4-nitrophenol. ACS Appl. Mater. Interfaces 13, 3209-3220.

Xiao, F., Qin, Y., Wang, N., Pan, D., 2018. Towards mass production of Au nanoparticles supported on montmorillonite microspheres for catalytic reduction of 4-nitrophenol. Appl. Clay Sci. 166, 74-79.

Xiao, F., Ren, H., Zhou, H., Wang, H., Wang, N., Pan, D., 2019. Porous montmorillonite@ graphene oxide@Au nanoparticle composite microspheres for organic dye degradation.ACS Appl. Nanostruct. Mater. 2, 5420-5429.

Xu, Y., Shi, X., Hua, R., Zhang, R., Yao, Y., Zhao, B., Liu, T., Zheng, J., Lu, G., 2020. Remarkably catalytic activity in reduction of 4-nitrophenol and methylene blue by $\mathrm{Fe}_{3} \mathrm{O}_{4} @ \mathrm{COF}$ supported noble metal nanoparticles. Appl. Catal. B 260, 118142.

Yang, W., Hu, W., Zhang, J., Wang, W., Cai, R., Pan, M., Huang, C., Chen, X., Yan, B., Zeng, H., 2021. Tannic acid/ $/ \mathrm{Fe}^{3+}$ functionalized magnetic graphene oxide nanocomposite with high loading of silver nanoparticles as ultra-efficient catalyst and disinfectant for wastewater treatment. Chem. Eng. J. 405, 126629.
Yang, Y., Jiang, K., Guo, J., Li, J., Peng, X., Hong, B., Wang, X., Ge, H., 2020. Facile fabrication of $\mathrm{Au} / \mathrm{Fe}_{3} \mathrm{O}_{4}$ nanocomposites as excellent nanocatalyst for ultrafast recyclable reduction of 4-nitropheol. Chem. Eng. J. 381, 122596.

Zhang, C., Cui, M., Ren, J., Xing, Y., Li, N., Zhao, H., Liu, P., Ji, X., Li, M., 2020a. Facile synthesis of novel spherical covalent organic frameworks integrated with Pt nanoparticles and multiwalled carbon nanotubes as electrochemical probe for tanshinol drug detection. Chem. Eng. J. 401, 126025.

Zhang, J., Yan, Z., Fu, L., Zhang, Y., Yang, H., Ouyang, J., Chen, D., 2018. Silver nanoparticles assembled on modified sepiolite nanofibers for enhanced catalytic reduction of 4-nitrophenol. Appl. Clay Sci. 166, 166-173.

Zhang, P., Wang, F., Qin, Y., Wang, N., 2020b. Exfoliated graphitic carbon nitride nanosheets/gold nanoparticles/spherical montmorillonite ternary porous heterostructures for the degradation of organic dyes. ACS Appl. Nano. Mater. 3 7847-7857.

Zhang, Q.P., Sun, Y.L., Cheng, G., Wang, Z., Ma, H., Ding, S.Y., Tan, B., Bu, J.H., Zhang, C., 2020c. Highly dispersed gold nanoparticles anchoring on post-modified covalent organic framework for catalytic application. Chem. Eng. J. 391, 123471.

Zhang, Y., Zhu, P., Chen, L., Li, G., Zhou, F., Lu, D.D., Sun, R., Zhou, F., Wong, C.P., 2014. Hierarchical architectures of monodisperse porous $\mathrm{Cu}$ microspheres: synthesis, growth mechanism, high-efficiency and recyclable catalytic performance. J. Mater. Chem. A 2, 11966-11973. 